Springer Link

\title{
Assessment of Cyclone Idai Floods on Local Food Systems and Disaster Management Responses in Mozambique and Zimbabwe
}

\author{
Cyclones in Southern Africa pp 59-68 | Cite as
}

- Daniel Tevera (1) Email author (dtevera@uwc.ac.za)

- Melusi Sibanda (2)

- Sipho Felix Mamba (3)

- Lucas Daniel Tivana (4)

1. Department of Geography, Environmental Studies and Tourism, University of the Western Cape, , Bellville, South Africa

2. Department of Agriculture, University of Zululand, , Richards Bay, South Africa

3. Faculty of Science and Engineering, Department of Geography, Environmental Science and Planning, University of Eswatini, , Kwaluseni, Eswatini

4. Faculty of Agronomy and Forest Engineering, Eduardo Mondlane University, , Maputo,

Mozambique

Chapter

First Online: 29 September 2021

Part of the Sustainable Development Goals Series book series (SDGS)

\begin{abstract}
In recent years, countries in southern Africa have experienced frequent hydro-meteorological disasters, such as widespread flooding caused by tropical cyclones. This chapter takes a close look at the destructive aspects of tropical cyclone Idai in Mozambique and Zimbabwe and the emergency disaster management responses. The chapter also seeks to understand the impact of the cyclone on food systems. The chapter is based on a desktop study that made use of scholarly publications and various media and organisation reports as the main sources of secondary data. A key finding of the study is that as the cyclone swept across the two countries, it exposed the fragilities of the local food systems, thereby presenting food insecurity challenges that potentially undermined the drive towards the achievement of Sustainable Development Goal (SDG) 2 on hunger eradication. The other finding is that the disaster management responses in both countries focussed on the emergency needs in the affected areas without giving much attention to making the food systems more resilient.
\end{abstract}

\section{Keywords}

Cyclone Idai Displacement Livelihoods Food systems SDG-2

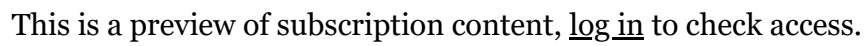

\section{Notes}

\section{Conflicts of Interest}

The authors declare no conflict of interest. 


\section{References}

Abrams, J. F., \& Abrams, A. L. (2018). Climate change challenges for Africa. Encyclopaedia of the Anthropocene, 2, 177-194.

CrossRef (https://doi.org/10.1016/B978-0-12-809665-9.09754-8)

Google Scholar (http://scholar.google.com/scholar_lookup?

title=Climate\%20change\%20challenges\%2ofor\%20Africa\&author=JF.\%2oAbrams\&author=AL.\% 20Abrams\&journal=Encyclopaedia\%20of\%20the\%20Anthropocene\&volume=2\&pages=177-

194\&publication_year=2018)

Akukwe, T. I., Oluoko-Odingo, A., \& Krhoda, G. O. (2020). Do floods affect food security? A before-and-after comparative study of flood-affected households' food security status in southeastern Nigeria. Bulletin of Geography Socio-Economic Series, 47(47), 115-131.

CrossRef (https://doi.org/10.2478/bog-2020-0007)

Google Scholar (http://scholar.google.com/scholar_lookup?

title=Do\%2ofloods\%20affect\%2ofood\%20security\%3F\%20A\%2obefore-and-

after\%20comparative\%20study\%20of\%2oflood-

affected\%2ohouseholds\%E2\%80\%99\%2ofood\%20security\%20status\%2oin\%20southeastern\%20Nigeria\&author=TI.\%20Akukwe\&author=A.\%20Oluoko-

Odingo\&author=GO.\%20Krhoda\&journal=Bulletin\%20of\%20Geography\%2oSocio-

Economic\%20Series\&volume $=47 \&$ issue $=47 \&$ pages $=115$-131\&publication_year $=2020$ )

Alhassan, H. (2020). Farm households' flood adaptation practices, resilience and food security in the Upper East region, Ghana. Heliyon, 6(1). https://doi.org/10.1016/j.heliyon.2020.e04167 (https://doi.org/10.1016/j.heliyon.2020.e04167)

Alam, E. (2017). Food security and household coping strategies during disasters in Bangladesh. International Journal of Natural Disasters \& Health Security, 3(1), 30-35.

Google Scholar (http://scholar.google.com/scholar_lookup?

title=Food\%20security\%2oand\%2ohousehold\%2ocoping\%2ostrategies\%2oduring\%2odisasters\% 20in\%2oBangladesh\&author=E.\%20Alam\&journal=International\%20Journal\%20of\%2oNatural \%20Disasters\%20\%26\%20Health\%20Security\&volume=3\&issue=1\&pages=30-

35\&publication_year=2017)

Burton, P., Lyons, K., Richards, C., Amati, M., Rose, N., Desfours, L., ... Barclay, R. (2013). Urban food security, urban resilience and climate change. National Climate Change Adaptation Research Facility (NCCARF).

Google Scholar (http://scholar.google.com/scholar_lookup?

title=Urban\%2 0 food\%20security\%2C\%20urban\%2oresilience\%20and\%2oclimate\%20change\&au thor=P.\%20Burton\&author=K.\%20Lyons\&author=C.\%20Richards\&author=M.\%20Amati\&author $=\mathrm{N} . \% 20$ Rose\&author=L.\%20Desfours\&publication_year=2013)

Chatiza, K. (2019). Cyclone Idai in Zimbabwe. An analysis of policy implications for post-disaster institutional development to strengthen disaster risk management. A report commissioned by Oxfam in Zimbabwe. Available from:

https://oxfamilibrary.openrepository.com/bitstream/handle/10546/620892/bp-impactresponse-cyclone-idai-zimbabwe-071119-en.pdf

(https://oxfamilibrary.openrepository.com/bitstream/handle/10546/620892/bp-impactresponse-cyclone-idai-zimbabwe-071119-en.pdf). Accessed 5 Oct 2020.

DW. (2020). Beira: Beira: Much to do a year after Cyclone Idai. March 15th 2020. Available on: https://www.dw.com/pt-002/beira-muito-a-fazer-um-ano-depois-do-ciclone-idai/a-52781102 (https://www.dw.com/pt-002/beira-muito-a-fazer-um-ano-depois-do-ciclone-idai/a-52781102). Accessed 25 June 2020.

Eisenhammer, S (2019). Hunger stalks Mozambique after deadly cyclone destroys farmland, environment, 1 April. Available from https://www.reuters.com/article/us-africa-cyclone-farmersidUSKCN1RD24Q (https://www.reuters.com/article/us-africa-cyclone-farmersidUSKCN1RD24Q). Accessed 25 June 2020.

FAO. (2015). The impact of natural hazards and disasters on agriculture and food security and nutrition: A call for action to build resilient livelihoods, May 2015. Available from:

http://www.fao.org/3/a-i4434e.pdf (http://www.fao.org/3/a-i4434e.pdf). Accessed 3 Dec 2020. 
FAO. (2019). Mozambique-cyclones Idai and Kenneth-Situation report-October 2019. Available from: on http://www.fao.org/3/ca7638en/ca7638en.pdf (http://www.fao.org/3/ca7638en/ca7638en.pdf). Accessed 5 July 2020.

IFPRI. (2019). Cyclone Idai, flooding, and food security in Malawi. Available from: https://www.ifpri.org/blog/cyclone-idai-flooding-and-food-security-malawi (https://www.ifpri.org/blog/cyclone-idai-flooding-and-food-security-malawi). Accessed 5 Sept 2020 .

INGC. (2019). Relatório de Avaliacao: Cyclone Idai. Available from: https://www.humanitarianresponse.info/sites/www.humanitarianresponse.info/files/assessments/beira_rapid_assessment_report_pt.pd (https://www.humanitarianresponse.info/sites/www.humanitarianresponse.info/files/assessmen ts/beira_rapid_assessment_report_pt.pdf). Accessed18 June 2020.

IPCC. (2014). Regional aspects (Africa). In: Climate Change 2014: Impacts, adaptation and vulnerability. Contribution of working group II to the fourth assessment report of the Intergovernmental Panel on Climate Change, 1, pp. 1-115. Available on http://ipccwg2.gov/AR5/report/final-drafts/ (http://ipcc-wg2.gov/AR5/report/final-drafts/). Accessed 5 Oct 2020.

Kiran, P., \& Madhumita, P. (2019). Agriculture, food security and livestock in southern Africa most vulnerable to climate hazards: FAO. Available from:

https://www.downtoearth.org.in/news/climate-change/agriculture-food-security-and-livestockin-southern-africa-most-vulnerable-to-climate-hazards-fao-65895

(https://www.downtoearth.org.in/news/climate-change/agriculture-food-security-and-livestockin-southern-africa-most-vulnerable-to-climate-hazards-fao-65895). Accessed 24 June 2020.

OCHA. (2019a). Southern Africa: Tropical Cyclone Kenneth. Flash update no 10. May 6th 2019.

Available from:

https://reliefweb.int/sites/reliefweb.int/files/resources/nationalsitrep_2_mozambique_17_may_2019_port.pdf (https://reliefweb.int/sites/reliefweb.int/files/resources/nationalsitrep_2_mozambique_17_may _2019_port.pdf). Accessed 7 July 2020.

OCHA. (2019b). Southern Africa: Tropical Cyclone Kenneth Flash Update No. 1212 May 2019. Available from https://reliefweb.int/sites/reliefweb.int/files/resources/Mozambique20190512_Flash\%20Update\%20\%2313.pdf (https://reliefweb.int/sites/reliefweb.int/files/resources/Mozambique20190512_Flash\%20Updat e\%20\%2313.pdf). Accessed 24 June 2020.

Pacetti, T., Caporali, E., \& Rulli, M. C. (2017). Floods and food security: A method to estimate the effect of inundation on crops availability. Advances in Water Resources, 11O(1), 494-504.

CrossRef (https://doi.org/10.1016/j.advwatres.2017.06.019)

Google Scholar (http://scholar.google.com/scholar_lookup?

title=Floods\%20and\%2ofood\%20security\%3A\%20A\%2omethod\%20to\%2oestimate\%20the\%2oef fect\%20of\%2oinundation\%20on\%20crops\%20availability\&author=T.\%20Pacetti\&author=E.\%20 Caporali\&author=MC.\%20Rulli\&journal=Advances\%20in\%20Water\%20Resources\&volume $=110$ \&issue $=1 \&$ pages $=494-504$ \&publication_year $=2017$ )

Pingali, P., Alinovi, L., \& Sutton, J. (2005). Food security in complex emergencies: Enhancing food system resilience. Disasters, 29, $\mathrm{S}_{5}-\mathrm{S} 24$.

CrossRef (https://doi.org/10.1111/j.0361-3666.2005.00282.x)

Google Scholar (http://scholar.google.com/scholar_lookup?

title=Food\%20security\%20in\%20complex\%20emergencies\%3A\%2oEnhancing\%2ofood\%20syste m\%20resilience\&author=P.\%20Pingali\&author=L.\%20Alinovi\&author=J.\%20Sutton\&journal=Di sasters\&volume $=29 \&$ pages $=\mathrm{S} 5$-S24\&publication_year $=2005$ )

Rakotobe, Z. L., Harvey, C. A., Rao, N. S., et al. (2016). Strategies of smallholder farmers for coping with the impacts of cyclones: A case study from Madagascar. International Journal of Disaster

Risk Reduction, 17, 114-122.

CrossRef (https://doi.org/10.1016/j.ijdrr.2016.04.013)

Google Scholar (http://scholar.google.com/scholar_lookup?

title=Strategies\%20of\%20smallholder\%2ofarmers\%2ofor\%2ocoping\%20with\%2othe\%2oimpacts \%20of\%20cyclones\%3A\%20A\%2ocase\%2ostudy\%2ofrom\%20Madagascar\&author=ZL.\%20Rakot obe\&author=CA.\%20Harvey\&author=NS.\%20Rao\&journal=International\%20Journal\%20of\%20 Disaster\%20Risk\%2oReduction\&volume=17\&pages=114-122\&publication_year=2016) 
Reason, C. J., \& Keibel, A. (2004). Tropical cyclone Eline and its unusual penetration and impacts over the southern African mainland. Weather and Forecasting, 19, 789-805.

CrossRef (https://doi.org/10.1175/1520-0434(2004)019<0789\%3ATCEAIU>2.0.CO\%3B2)

Google Scholar (http://scholar.google.com/scholar_lookup?

title=Tropical\%20cyclone\%20Eline\%20and\%20its\%20unusual\%2openetration\%20and\%20impac ts\%20over\%20the\%20southern\%20African\%2omainland\&author=CJ.\%2OReason\&author=A.\%2 oKeibel\&journal=Weather\%20and\%20Forecasting\&volume $=19 \&$ pages $=789$ -

805\&publication_year=2004)

Ringler, C., Zhu, T., Cai, X., Koo, J., \& Wang, D. (2010). Climate Change impacts on food security in Sub-Saharan Africa insights from comprehensive Climate Change scenarios. Food Policy, 466(28). https://doi.org/10.1038/natureo9146 (https://doi.org/10.1038/natureo9146)

Salazar-Espinoza, C., Jones, S., \& Tarp, F. (2015). Weather shocks and cropland decisions in rural Mozambique. Food Policy, 53, 9-21.

CrossRef (https://doi.org/10.1016/j.foodpol.2015.03.003)

Google Scholar (http://scholar.google.com/scholar_lookup?

title=Weather\%20shocks\%20and\%20cropland\%20decisions\%20in\%20rural\%20Mozambique\&au thor $=$ C.\%2oSalazar-

Espinoza\&author=S.\%20Jones\&author=F.\%20Tarp\&journal=Food\%20Policy\&volume=53\&pages $=9-21 \&$ publication_year=2015)

TransConflict. (2019). The impact of Cyclone Idai - Lessons for Africa. Available from http://www.transconflict.com/2019/o6/the-impact-of-cyclone-idai-lessons-for-africa/ (http://www.transconflict.com/2019/06/the-impact-of-cyclone-idai-lessons-for-africa/). Accessed 24 June 2020.

UNEP (United Nations Environment Programme) and SARDC (Southern African Research and Documentation Centre). (2009). Droughts and floods in southern Africa. Environmental change and human vulnerability. UNEP and SARDC.

Google Scholar (http://scholar.google.com/scholar_lookup?

title=Droughts\%20and\%2ofloods\%20in\%20southern\%20Africa.\%2oEnvironmental\%2ochange\% 20and\%20human\%20vulnerability\&publication_year=2009)

UNICEF. (2019). Mozambique: Children living in storm-affected areas face worsening food insecurity and nutrition crisis six months after cyclone Idai. September 14th 2019.

Google Scholar (https://scholar.google.com/scholar?

q=UNICEF.\%20\%282019\%29.\%20Mozambique\%3A\%20Children\%2oliving\%2oin\%20stormaffected\%20areas\%2oface\%20worsening\%2ofood\%2oinsecurity\%20and\%2onutrition\%2ocrisis\% 20six\%20months\%20after\%20cyclone\%20Idai.\%20September\%2014th\%202019.)

Week, D. A., \& Wizor, C. H. (2020). Effects of flood on food security, livelihood and socioeconomic characteristics in the flood in the flood-prone areas of the Core Niger Delta, Nigeria. Asian Journal of Geographical Research, 3(1), 1-17.

CrossRef (https://doi.org/10.9734/ajgr/2020/v3i130096)

Google Scholar (http://scholar.google.com/scholar_lookup?

title=Effects\%2Oof\%2Oflood\%20on\%2ofood\%2osecurity\%2C\%2olivelihood\%20and\%20socioeconomic\%20characteristics\%20in\%20the\%20flood\%20in\%20the\%2oflood-

prone\%20areas\%20of\%20the\%20Core\%20Niger\%20Delta\%2C\%20Nigeria\&author=DA.\%2oWee k\&author=CH.\%2OWizor\&journal=Asian\%2OJournal\%20of\%2oGeographical\%20Research\&volu $\mathrm{me}=3$ \&issue $=1 \&$ pages $=1-17$ \&publication_year $=2020$ )

World Bank. (2019). Mozambique: Cyclone Idai \& Kenneth Emergency Recovery and Resilience Project (P17104O). Available on http://documents1.worldbank.org/curated/en/

(http://documents1.worldbank.org/curated/en/) 727131568020768626/pdf/Project-

Information-Document-Mozambique-Cyclone-Idai-Kenneth-Emergency-Recovery-and-

Resilience-Project-P171040.pdf. Accessed 20 Jan 2020.

World Food Programme. (2018). The State of Food Insecurity and Nutrition in the World.

Available at http://www.fao.org/3/i9553en/i9553en.pdf

(http://www.fao.org/3/i9553en/i9553en.pdf). Accessed 20 Jan 2021.

World Food Programme. (2019). Food Security in Southern Africa in 2019/20. Available on https://docs.wfp.org/api/documents/WFP-0000106747/download/?iframe

(https://docs.wfp.org/api/documents/WFP-0000106747/download/?iframe). Accessed 20 Jan 2020. 
World Vision. (2020). 2019 Cyclone Idai: Facts, FAQs, and how to help. Available from https://www.worldvision.org/disaster-relief-news-stories/2019-cyclone-idai-facts\#damage (https://www.worldvision.org/disaster-relief-news-stories/2019-cyclone-idai-facts\#damage). Accessed 24 June 2020.

\section{Copyright information}

(C) The Author(s), under exclusive license to Springer Nature Switzerland AG 2021

\section{About this chapter}

Cite this chapter as:

Tevera D., Sibanda M., Mamba S.F., Tivana L.D. (2021) Assessment of Cyclone Idai Floods on Local Food Systems and Disaster Management Responses in Mozambique and Zimbabwe. In: Nhamo G., Chikodzi D. (eds) Cyclones in Southern Africa. Sustainable Development Goals Series. Springer, Cham. https://doi.org/10.1007/978-3-030-74303-1_4

- First Online 29 September 2021

- DOI https://doi.org/10.1007/978-3-030-74303-1_4

- Publisher Name Springer, Cham

- Print ISBN 978-3-030-74302-4

- Online ISBN 978-3-030-74303-1

- eBook Packages Earth and Environmental Science Earth and Environmental Science (Ro).

- Buy this book on publisher's site

- Reprints and Permissions

\section{Personalised recommendations}

\section{SPRINGER NATURE}

(C) 2020 Springer Nature Switzerland AG. Part of $\underline{\text { Springer Nature. }}$

Not logged in Not affiliated 102.249.3.157 